\title{
Trait emotional experience in individuals with schizophrenia and youth at clinical high risk for psychosis
}

\author{
Claire I. Yee, Gregory P. Strauss, Daniel N. Allen, Claudia M. Haase, David Kimhy and Vijay A. Mittal
}

\section{Background}

Disturbances in trait emotions are a predominant feature in schizophrenia. However, less is known about (a) differences in trait emotion across phases of the illness such as the clinical high-risk (CHR) phase and (b) whether abnormalities in trait emotion that are associated with negative symptoms are driven by primary (i.e. idiopathic) or secondary (e.g. depression, anxiety) factors.

\section{Aims}

To examine profiles of trait affective disturbance and their clinical correlates in individuals with schizophrenia and individuals at CHR for psychosis.

\section{Method}

In two studies (sample 1: 56 out-patients diagnosed with schizophrenia and 34 demographically matched individuals without schizophrenia (controls); sample 2: 50 individuals at CHR and 56 individuals not at CHR (controls)), participants completed self-report trait positive affect and negative affect questionnaires, clinical symptom interviews (positive, negative, disorganised, depression, anxiety) and community-based functional outcome measures.

\section{Results}

Both clinical groups reported lower levels of positive affect (specific to joy among individuals with schizophrenia) and higher levels of negative affect compared with controls. For individuals with schizophrenia, links were found between positive affect and negative symptoms (which remained after controlling for secondary factors) and between negative affect and positive symptoms. For individuals at CHR, links were found between both affect dimensions and both types of symptom (which were largely accounted for by secondary factors).

\section{Conclusions}

Both clinical groups showed some evidence of reduced trait positive affect and elevated trait negative affect, suggesting that increasing trait positive affect and reducing trait negative affect is an important treatment goal across both populations. Clinical correlates of these emotional abnormalities were more integrally linked to clinical symptoms in individuals with schizophrenia and more closely linked to secondary influences such as depression and anxiety in individuals at CHR.

\section{Declaration of interest}

None.

\section{Keywords}

Psychosis; prodrome; emotion; negative symptoms.

\section{Copyright and usage}

(c) The Royal College of Psychiatrists 2019. This is an Open Access article, distributed under the terms of the Creative Commons Attribution licence (http://creativecommons.org/licenses/by/4.0/), which permits unrestricted re-use, distribution, and reproduction in any medium, provided the original work is properly cited.
Emotional abnormalities have been a core aspect of schizophrenia since the earliest conceptualisations of the disorder. For example, Kraepelin described individuals with schizophrenia as having 'a weakening of those emotional activities which permanently form the mainsprings of volition'. ${ }^{1}$ Bleuler similarly emphasised that individuals with schizophrenia were 'disturbed in a number of ways, but above all the breakdown of the emotion'. ${ }^{1}$ Modern empirical studies support these early observations by documenting abnormalities in a variety of emotional processes, such as facial and vocal expressivity, emotion perception, emotion regulation and trait emotional experience; ${ }^{2-6}$ however, some emotional processes appear to be intact (e.g. state positive emotional experience). ${ }^{7-9}$ Importantly, when abnormalities are present in the aforementioned processes, they are consistently linked to poor clinical outcomes, such as increased severity of positive, negative and disorganised symptoms, poor community-based functional outcome and lower quality of life. ${ }^{10-14}$

Although emotional abnormalities are clinically important in the active phases of schizophrenia, it is unclear whether they are also associated with liability for the illness. Psychotic disorders are typically preceded by a prodromal (i.e. pre-illness) phase characterised by functional decline and subtle attenuated hallucinations and delusions that progressively worsen over the course of several months to years. ${ }^{15,16}$ This period is of interest both as a window for investigating processes involved in illness onset and as a potential point of intervention and prevention. ${ }^{17,18}$ Documenting the nature of emotional abnormalities among individuals at clinical high risk (CHR) for psychosis is critical for enhancing early identification and intervention efforts. ${ }^{19,20}$

\section{Trait emotional experience studies in schizophrenia}

Trait emotional experience (i.e. dispositional tendencies to experience positive or negative emotions in response to life's events in enduring and predictable ways ${ }^{21}$ ) is a critical aspect of emotionality that has received relatively little attention in the CHR literature. Studies administering trait emotional experience questionnaires to healthy individuals typically identify two distinct dimensions that are only moderately correlated: positive and negative affect. ${ }^{22}$ These dimensions predict mental and physical health for a number of conditions. ${ }^{23,24}$ A large number of studies administering trait questionnaires to individuals with schizophrenia indicate that they report lower levels of general positive affect and higher levels of general negative affect than individuals without schizophrenia (for a review see Horan et $a l^{11}$ ). Differences in discrete trait emotions can also be observed between individuals with schizophrenia and those without. For example, individuals with schizophrenia report lower levels of trait joy and higher levels of trait fear and disgust and these differences are greatest for those with high levels of anhedonia and blunted affect. ${ }^{25}$ In a more general vein, low trait positive affect in schizophrenia has been linked to lower quality of life, smaller 
social networks, depression, anxiety and anhedonia. ${ }^{26-28}$ The association between lower levels of positive affect and negative symptoms is particularly strong across studies. ${ }^{29-32}$ Conversely, high trait negative affect in schizophrenia has been linked to worse coping, occupational and social functioning, lower satisfaction with life, as well as increased smoking, alcohol and drug use. ${ }^{28,33-36}$

To our knowledge, only one study has evaluated trait positive and negative affect in individuals at CHR for psychosis. Seo and colleagues $^{37}$ found that individuals at CHR reported lower levels of trait positive and higher levels of trait negative affect. Moreover, only negative symptoms predicted individual differences in both higher negative and lower positive trait affect.

Taken together, these findings suggest that trait affect may be critically related to negative symptoms in both individuals with schizophrenia and individuals at CHR for psychosis. However, negative symptoms are heterogeneous and it is possible for two participants to receive exactly the same score on a rating scale for different reasons. In schizophrenia, it is well-documented that negative symptoms can reflect both primary (i.e. idiopathic) and secondary (e.g. depression, anxiety) factors. ${ }^{38}$ Individuals with schizophrenia with primary and enduring negative symptoms have clinical, biological and emotional profiles that are distinct from individuals with secondary negative symptoms or those with low levels of negative symptoms. ${ }^{39-42}$ Secondary negative symptom sources (e.g. depression, anxiety) are also prominent in individuals at $\mathrm{CHR}^{43}$ and are strongly associated with negative symptoms; ${ }^{44}$ however, secondary negative symptoms tend to be transient, resolving with alleviation of the secondary influence. In contrast, primary negative symptoms tend to be persistent during the prodromal period, and can be identified at rates comparable to those in chronic schizophrenia $(\sim 33 \%){ }^{45}$ These findings suggest that clarifying the extent to which trait affective abnormalities are linked to primary and secondary negative symptoms across phases of illness is of critical importance because it would suggest how treatment efforts would be best directed at each stage.

\section{The current study}

The current study administered a trait emotional experience questionnaire and measures of clinical outcomes to cross-sectional samples of out-patients with schizophrenia and at CHR for psychosis to examine profiles of trait affective disturbance and their clinical correlates. The following hypotheses were tested: (a) both individuals with schizophrenia and those at CHR for psychosis would evidence diminished trait positive affect and elevated trait negative affect compared with control groups; (b) lower trait positive affect would be associated with increased severity of negative symptoms and poorer community-based functional outcome across both the sample with schizophrenia and the sample at CHR; and (c) higher trait negative affect would be associated with increased severity of positive and negative symptoms and poorer community-based functioning across both the samples with schizophrenia and the sample at CHR.

Prior psychosis studies have administered measures that were not amenable to examining the role of discrete emotions; however, examination of discrete emotions is an important extension because individual positive (e.g. joy, interest) and negative (e.g. sadness, fear) emotions can have unique effects on physiology, health and behaviour. ${ }^{46-48}$ We therefore conducted exploratory analyses to determine whether individuals with schizophrenia and those at CHR showed different patterns of abnormalities on discrete trait emotions. Exploratory analyses were also conducted to determine whether associations between negative symptoms and trait affect reflected secondary factors in the samples with schizophrenia and at CHR. These analyses focused on depression, anxiety and antipsychotics as the most common secondary factors that are prevalent in both populations with schizophrenia and those at CHR. ${ }^{38,43}$ Negative symptoms do not reflect a singular construct, ${ }^{49}$ but rather two broad dimensions reflecting diminished expressivity and diminished motivation and pleasure, or five discrete domains reflecting anhedonia, avolition, asociality, alogia and blunted affect. ${ }^{50-52}$ Therefore, exploratory analyses examined associations between trait affect and the two broad negative symptom dimensions, as well as the five domains.

\section{Method}

The samples used for the study originate from two separate studies. Both studies were approved by the local institutional review boards, and written informed consent or assent was obtained from all participants.

\section{Sample 1}

\section{Participants}

Sample 1 comprised 56 individuals meeting DSM-IV-TR ${ }^{53}$ criteria for schizophrenia (79\%) or schizoaffective disorder (21\%) and 34 individuals without schizophrenia (controls). Individuals with schizophrenia were recruited from out-patient psychiatric mental health facilities and evaluated during periods of clinical stability, as determined by a minimum of 4 weeks of consistent medication dose and type or, in the absence of medication, deemed clinically stable by their treating clinician, as indicated by no significant changes in symptoms or functioning within the previous month. Consensus diagnosis was established via a best-estimate approach based on psychiatric history and multiple interview and subsequently confirmed using the Structured Clinical Interview for DSM-IV (SCID-I $\left.{ }^{54}\right)$. All individuals with schizophrenia met DSM-IV current diagnostic criteria for schizophrenia or schizoaffective disorder; $93 \%$ were prescribed antipsychotic medications at the time of testing. Individuals without schizophrenia were recruited through posted advertisements and word of mouth among enrolled participants. All individuals without schizophrenia underwent a screening interview, including the SCID-I, and did not meet lifetime criteria for a psychotic disorder or any current Axis I disorder. Individuals without schizophrenia also had no selfreported family history of psychosis. The SCID-I was used to determine that no participants met DSM-IV criteria for substance misuse or dependence over the previous 6 months. All participants were also screened for lifetime neurological disorders and were free from neurological conditions (e.g. traumatic brain injury, epilepsy). Demographic details for sample 1 are given in Table 1.

\section{Measures}

Clinical interviews. The Scale for the Assessment of Positive Symptoms (SAPS) ${ }^{55}$ and Negative Symptoms (SANS) ${ }^{56}$ were administered by clinical psychology doctoral students trained to reliability standards $(\alpha>0.80)$ to measure participants' positive and negative symptoms. The Brief Psychiatric Rating Scale (BPRS) ${ }^{57}$ was used to assess anxiety and depression. The Global Assessment of Functioning (GAF) score was used as a gross measure of global functioning.

Trait emotional experience. The Differential Emotions Scale IV-A $(\mathrm{DES})^{58}$ is a 30 -item self-report measure designed to assess the frequency with which individuals experience discrete basic emotions in everyday life. The questionnaire yields ten basic emotion scales (each measured by three items), with three scales representing positive emotions (joy, interest and surprise), which are averaged to measure positive affect $(\alpha=0.73)$, and seven representing negative 


\begin{tabular}{|c|c|c|c|}
\hline & Individuals with SZ & Individuals without SZ & Total \\
\hline Age, mean (s.d.) & $39.30(9.72)$ & $37.79(12.43)$ & $38.73(10.78)$ \\
\hline \multicolumn{4}{|l|}{ Gender, $n$} \\
\hline Male & 30 & 13 & 43 \\
\hline Female & 26 & 21 & 47 \\
\hline Total & 56 & 34 & 90 \\
\hline Education, years: mean (s.d.) & $11.88(1.99)$ & $13.60(1.42)$ & $12.53(1.98)$ \\
\hline Antipsychotics, in CPZeq/day: mean (s.d.) & $773.76(637.10)$ & & \\
\hline DES positive affect, mean (s.d.) & $1.05(0.25)$ & $1.05(0.15)$ & $1.05(0.22)$ \\
\hline DES negative affect, mean (s.d.) & $0.85(0.27)$ & $0.70(0.19)$ & $0.80(0.26)$ \\
\hline BPRS anxiety, mean (s.d.) & $3.18(1.58)$ & - & - \\
\hline BPRS depression, mean (s.d.) & $1.89(1.45)$ & - & - \\
\hline GAF, mean (s.d.) & $31.94(11.62)$ & - & - \\
\hline SAPS, mean (s.d.) ${ }^{a}$ & $33.72(18.68)$ & - & - \\
\hline SANS, mean (s.d.) ${ }^{\mathrm{a}}$ & $43.71(28.45)$ & - & - \\
\hline Diminished expression, mean (s.d.) & $1.22(1.06)$ & - & - \\
\hline Diminished motivation/pleasure, mean (s.d.) & $1.50(1.17)$ & - & - \\
\hline Blunted affect, mean (s.d.) & $1.32(1.24)$ & - & - \\
\hline Alogia, mean (s.d.) & $1.11(1.09)$ & - & - \\
\hline Avolition, mean (s.d.) & $1.4(1.11)$ & - & - \\
\hline Anhedonia, mean (s.d.) & $1.34(1.37)$ & - & - \\
\hline Asociality, mean (s.d.) & $1.75(1.43)$ & - & - \\
\hline
\end{tabular}

emotions (sadness, anger, fear, disgust, contempt, guilt and shame), which are averaged to measure negative affect $(\alpha=0.91)$. Participants are required to identify the frequency with which they experience 30 emotions on a 5 -point scale (1, rarely or never experienced; 5, experienced very often). DES items reflect verbal labels commonly applied to identify facial expressions, as well as current theories of basic emotion, which describe emotions in terms of discrete categories. ${ }^{59}$ This measure has been used in previous schizophrenia investigations, and demonstrated differences in experience among discrete emotions in individuals with and without schizophrenia. ${ }^{25}$

\section{Sample 2}

Participants

Sample 2 comprised a group of 50 adolescents and young adults aged between 13 and 21 years (mean 19.06, s.d. $=2.28$ ) at CHR for psychosis and 56 matched individuals not at CHR (controls) recruited to the Adolescent Development and Preventive Treatment (ADAPT) research programme using internet, newspaper and public transportation advertisements, email postings and community professional referrals. Individuals at CHR in the present study met Structured Interview for Prodromal Syndromes (SIPS) criteria for a prodromal syndrome, defined by moderateto-severe but not psychotic levels of positive symptoms (rated between 3 and 5 on a 6-point scale) or a decline in global functioning accompanying the presence of schizotypal personality disorder or a family history of schizophrenia. ${ }^{60}$ Family history of psychosis was obtained by asking participants whether any first-degree family members had been diagnosed with a psychotic disorder. In most cases, family history was corroborated by another family member of the participant. Exclusion criteria consisted of head injury, the presence of a neurological disorder and lifetime substance dependence. The presence or lifetime history of an Axis I psychotic disorder at baseline was also an exclusion criterion for control and CHR status. See Table 2 for demographic information.

\section{Table 2 Demographic details for sample 2}

\begin{tabular}{|c|c|c|c|}
\hline & Individuals at $\mathrm{CHR}$ & Individuals not at $\mathrm{CHR}$ & Total \\
\hline Age, mean (s.d.) & $18.94(1.82)$ & $19.16(2.62)$ & $19.06(2.28)$ \\
\hline \multicolumn{4}{|l|}{ Gender, $n$} \\
\hline Male & 29 & 22 & 51 \\
\hline Female & 21 & 34 & 55 \\
\hline Total & 50 & 56 & 106 \\
\hline Parental education, years: mean (s.d.) ${ }^{a}$ & $15.67(2.30)$ & $15.73(2.63)$ & $15.6(2.49)$ \\
\hline Participants taking antipsychotics, $n$ & 9 & 0 & 9 \\
\hline Positive affect, mean (s.d.) & $2.37(0.73)$ & $2.88(0.57)$ & $2.64(0.68)$ \\
\hline Negative affect, mean (s.d.) & $1.79(0.85)$ & $0.81(0.76)$ & $1.27(0.93)$ \\
\hline BAl, mean (s.d.) & $20.32(12.18)$ & $5.65(6.65)$ & $12.51(12.08)$ \\
\hline BDI, mean (s.d.) & $1.29(11.41)$ & $3.21(4.40)$ & $12.51(12.08)$ \\
\hline GFS: Social, mean (s.d.) & $6.88(1.65)$ & $8.73(0.68)$ & $7.86(1.53)$ \\
\hline GFS: Role, mean (s.d.) & $6.96(1.49)$ & $8.65(0.70)$ & $7.88(1.41)$ \\
\hline SIPS positive, mean (s.d.) ${ }^{\mathrm{b}}$ & $11.78(4.62)$ & $0.34(0.84)$ & $5.62(6.53)$ \\
\hline SIPS negative, mean (s.d.) $)^{b}$ & $9.58(7.37)$ & $0.23(0.54)$ & $4.52(6.83)$ \\
\hline Diminished emotion, mean (s.d.) & $1.78(1.51)$ & $0.05(0.14)$ & $0.84(1.34)$ \\
\hline Diminished volition, mean (s.d.) & $1.64(1.37)$ & $0.03(0.12)$ & $0.77(1.23)$ \\
\hline
\end{tabular}




\section{Measures}

Clinical interviews. Interviews were administered by expert raters trained to reliability standards $(\alpha>0.80)$. The SIPS was administered at baseline to diagnose a prodromal syndrome and assess symptoms. ${ }^{60,61}$ A total sum score for the positive, negative and disorganised symptom domains is used as an indicator of the respective dimensions of symptomatology.

Depression and anxiety were assessed using the Beck Depression Inventory (BDI) ${ }^{62}$ and the Beck Anxiety Inventory (BAI). ${ }^{63}$

The Global Functioning Scale: Social (GFS-S) and Role (GFS-R) ${ }^{64}$ were used to assess community-based functional outcome (e.g. number of friends, how often the individual engages in social activity, performance at school, work). A score is given on a 1-10 scale, with 1 indicating low levels of functioning and 10 indicating very high levels of functioning.

Trait emotional experience. The Modified Differential Emotions Scale $(\mathrm{mDES})^{65}$ was used to measure trait emotional experience. Participants rated the extent to which they felt each of 20 emotions on average or in general on a 5-point scale from 0 (not at all) to 4 (extremely). The mDES differs from the DES in that it measures each emotion with a single item, thus increasing the number of discrete positive emotions from three to ten. The ten positive emotions (amusement, awe, content, joy, gratitude, hope, interest, love, pride and surprise) were averaged together to form the positive affect composite $(\alpha=0.88)$. The negative affect composite averaged scores for anger, shame, fear, disgust, embarrassment, guilt, sadness and contempt $(\alpha=0.93)$.

\section{Data analytic strategy}

Repeated measures ANOVAs were used to test differences in the pattern of self-reported emotional experience among clinical and control samples. Each ANOVA had two groups (schizophrenia/ CHR, control) with two emotion dimensions (positive, negative). Significant interactions were followed up with one-way ANOVAs and within-group paired samples $t$-tests.

Correlations were analysed to examine the relationship between emotionality, symptoms and functioning in the individuals with schizophrenia and those at CHR. In the first set of correlations, emotionality included the positive and negative affect composites, symptoms included positive and negative totals (and disorganised for sample 2) and functionality included the GAF for sample 1 and GFS-S and GFS-R for sample 2. In the second set of correlations, negative symptoms were broken into two subsets: two subdimensions (i.e. diminished expression and diminished motivation in individuals with schizophrenia, and diminished emotion and diminished volition in individuals at CHR), ${ }^{66,67}$ and the five National Institute of Mental Health (NIMH) negative symptom domains (i.e. anhedonia, avolition, asociality, alogia and blunted affect). ${ }^{68}$ To test the extent to which relationships in this second set of correlations were influenced by secondary factors, partial correlations were run controlling for depression and anxiety (and antipsychotics in sample 1). In sample 2, all partial correlations were rerun excluding individuals on antipsychotics. No correlations changed significantly in magnitude or direction when excluding these individuals. The Benjamini-Hochberg correction procedure was used for each set of correlations to control for multiple correlation tests. ${ }^{69}$ Results of the procedure are stated at the end of each correlation section.

\section{Results}

\section{Group differences in trait emotional experience}

Affect differences between individuals with and without schizophrenia

A repeated-measures ANOVA was conducted to test for group differences in trait emotional experience between individuals with and without schizophrenia. There was a statistically significant group $\times$ emotion interaction, in addition to a main effect of group and a within-participants effect of emotion. Follow-up one-way ANOVAs showed higher levels of negative emotion in individuals with schizophrenia compared with individuals without schizophrenia, but no group differences in positive emotion. Paired-samples $t$-tests showed higher levels of positive than negative emotion within each group (Table 3; Fig. 1).

\section{Affect differences between individuals at $\mathrm{CHR}$ and not at $\mathrm{CHR}$}

A repeated-measures ANOVA showed a significant group $\times$ emotion interaction, in addition to a main effect of group and a within-participants effect of emotion. Follow-up one-way

Table 3 Omnibus ANOVA and post hoc results for group affect differences

Test statistic

P

$F_{1,80}=4.06$

Group

Emotion

Group $\times$ emotion

Post hoc one-way ANOVAs

Positive

Negative

Post hoc paired-samples $t$-tests

SZ: positive $v$. negative

Control: positive $v$. negative

Sample 2: positive $v$. negative affect

Group

Emotion

Group $\times$ emotion

Post hoc one-way ANOVAs

Positive

Negative

Post hoc paired-sample $t$-tests

CHR: positive $v$. negative

Control: positive $v$. negative
$F_{1,80}=55.28$

$F_{1,80}=4.06$

$F_{1,80}<0.001$

$F_{1,80}=7.42$

$t_{53}=4.27$

$t_{29}=6.66$

$F_{1,103}=7.10$

$F_{1,103}=137.05$

$F_{1,103}=43.00$

$F_{1,103}=16.38$

$F_{1,103}=38.31$

$t_{50}=3.04$

$t_{55}=16.26$
0.05

$<0.001$

0.04

0.99

$<0.01$

$<0.001$

$<0.001$

$<0.01$

$<0.001$

$<0.001$

$<0.001$

$<0.001$

$<0.01$

$<0.001$
Cohen's d

0.46
1.67
0.46

$<0.01$
0.64

0.59
1.24

0.55
2.30
1.31

0.80
1.22

0.43
2.19

SZ, individuals with schizophrenia; $\mathrm{CHR}$, individuals at clinical high risk for psychosis 

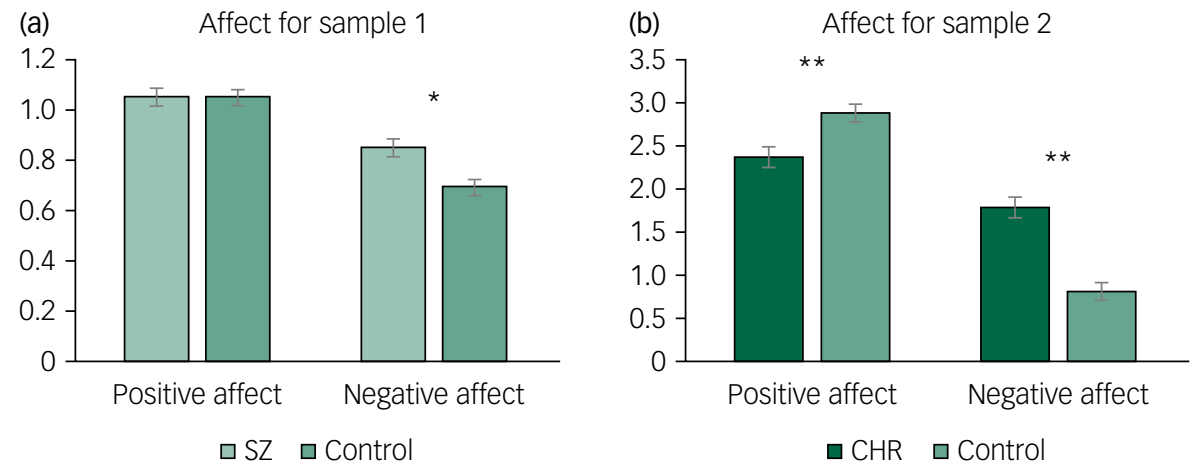

Fig. 1 Differences in positive and negative affect in samples 1 and 2 .

Bars in each graph represent standard error.

$\mathrm{SZ}$, individuals with schizophrenia; CHR, individuals at clinical high risk for psychosis.

ANOVAs showed lower levels of positive and higher levels of negative affect in individuals at CHR for psychosis compared with individuals not at CHR. Paired-samples $t$-tests showed higher levels of positive than negative emotion within each group (Table 3; Fig. 1).

\section{Exploratory discrete emotion differences between both samples}

Across samples, both repeated-measures ANOVAs showed a significant group $\times$ emotion interaction and a within-participants effect of emotion. Follow-up one-way ANOVAs showed that in sample 1, two of the three positive emotions (i.e. joy and surprise) showed significant differences between individuals with and without schizophrenia. For joy, individuals with schizophrenia reported lower joy than individuals without schizophrenia, whereas individuals with schizophrenia reported more surprise compared with individuals without schizophrenia. However, after correcting for multiple tests, these two differences between groups were not significant. Four of the eight negative emotions (i.e. shame, fear, disgust and sadness) showed marginal or significant differences (Table 4), whereas the other four (i.e. anger, guilt, contempt and hostility) showed no significant differences between individuals with and without schizophrenia. In sample 2, seven of the ten positive emotions (i.e. amusement, contentment, joy, gratitude, hope, love and pride) and each of the eight negative emotions (i.e. anger, shame, fear, disgust, embarrassment, guilt, sadness and contempt) showed significant differences between individuals at and not at CHR for psychosis (Fig. 2). In all cases, individuals at CHR reported more negative emotions than individuals not at $\mathrm{CHR}$. All group differences remained unchanged after correcting for multiple testing, except for the findings for joy and surprise in sample 1.

\section{Trait emotional experience and clinical and functional outcomes}

Affect relationships to symptoms and functioning in individuals with schizophrenia

Pearson correlations were conducted to investigate the degree to which positive and negative emotions were related to clinical symptoms and functional outcomes in the individuals with schizophrenia. Lower levels of positive affect were related to higher levels of negative symptoms but were not significantly related to positive symptoms. Higher levels of negative affect were related to higher levels of positive symptoms and were unrelated to negative symptoms. Neither type of emotion was directly related to functioning (Fig. 3). All relationships remained unchanged after correcting for multiple correlation tests.
Affect relationships to symptoms and functioning in individuals at CHR Correlations in the individuals at CHR for psychosis showed that lower levels of positive affect were related to higher levels of both positive and negative symptoms. Higher levels of negative affect were related to higher levels of positive, negative and disorganised

\begin{tabular}{|c|c|c|c|}
\hline & Test statistic & $P$ & Cohen's d \\
\hline \multicolumn{4}{|l|}{ Sample 1} \\
\hline Group & $F_{1,80}=6.61$ & 0.01 & 0.60 \\
\hline Emotion & $F_{10,800}=30.38$ & $<0.001$ & 1.29 \\
\hline Group × emotion & $F_{10,800}=4.94$ & $<0.01$ & 0.52 \\
\hline \multicolumn{4}{|c|}{ Post hoc one-way ANOVAs } \\
\hline Joy & $F_{1,80}=4.47$ & $0.04^{a}$ & 0.49 \\
\hline Interest & $F_{1,80}=0.12$ & 0.73 & 0.08 \\
\hline Surprise & $F_{1,80}=6.18$ & $0.02^{\mathrm{a}}$ & 0.58 \\
\hline Anger & $F_{1,80}=0.41$ & 0.52 & 0.15 \\
\hline Shame & $F_{1,80}=11.60$ & $<0.01$ & 0.80 \\
\hline Fear & $F_{1,80}=18.18$ & $<0.001$ & 1.00 \\
\hline Disgust & $F_{1,80}=9.15$ & $<0.01$ & 0.71 \\
\hline Guilt & $F_{1,80}=0.66$ & 0.42 & 0.19 \\
\hline Sadness & $F_{1,80}=3.81$ & 0.06 & 0.46 \\
\hline Contempt & $F_{1,80}=1.82$ & 0.18 & 0.32 \\
\hline Hostility & $F_{1,80}=2.44$ & 0.12 & 0.37 \\
\hline \multicolumn{4}{|l|}{ Sample 2} \\
\hline Group & $F_{1,103}=2.64$ & 0.11 & 0.32 \\
\hline Emotion & $F_{17,1751}=67.15$ & $<0.001$ & 1.62 \\
\hline Group × emotion & $F_{1,1751}=21.25$ & $<0.001$ & 0.91 \\
\hline \multicolumn{4}{|c|}{ Post hoc one-way ANOVAs } \\
\hline Amusement & $F_{1,103}=12.23$ & $<0.01$ & 0.69 \\
\hline Awe & $F_{1,103}=0.68$ & $=0.41$ & 0.16 \\
\hline Contentment & $F_{1,103}=2.64$ & $<0.01$ & 0.32 \\
\hline Joy & $F_{1,103}=30.47$ & $<0.001$ & 1.09 \\
\hline Gratitude & $F_{1,103}=19.75$ & $<0.001$ & 0.88 \\
\hline Hope & $F_{1,103}=20.65$ & $<0.001$ & 0.90 \\
\hline Interest & $F_{1,103}=1.71$ & $=0.19$ & 0.26 \\
\hline Love & $F_{1,103}=15.72$ & $<0.001$ & 0.78 \\
\hline Pride & $F_{1,103}=9.84$ & $<0.01$ & 0.62 \\
\hline Surprise & $F_{1,103}=.21$ & $=0.65$ & 0.09 \\
\hline Anger & $F_{1,103}=24.89$ & $<0.001$ & 0.98 \\
\hline Shame & $F_{1,103}=15.58$ & $<0.001$ & 0.78 \\
\hline Fear & $F_{1,103}=29.04$ & $<0.001$ & 1.06 \\
\hline Disgust & $F_{1,103}=17.38$ & $<0.001$ & 0.82 \\
\hline Embarrassment & $F_{1,103}=19.11$ & $<0.001$ & 0.86 \\
\hline Guilt & $F_{1,103}=28.20$ & $<0.001$ & 1.05 \\
\hline Sadness & $F_{1,103}=39.64$ & $<0.001$ & 1.24 \\
\hline Contempt & $F_{1,103}=17.58$ & $<0.001$ & 0.83 \\
\hline
\end{tabular}



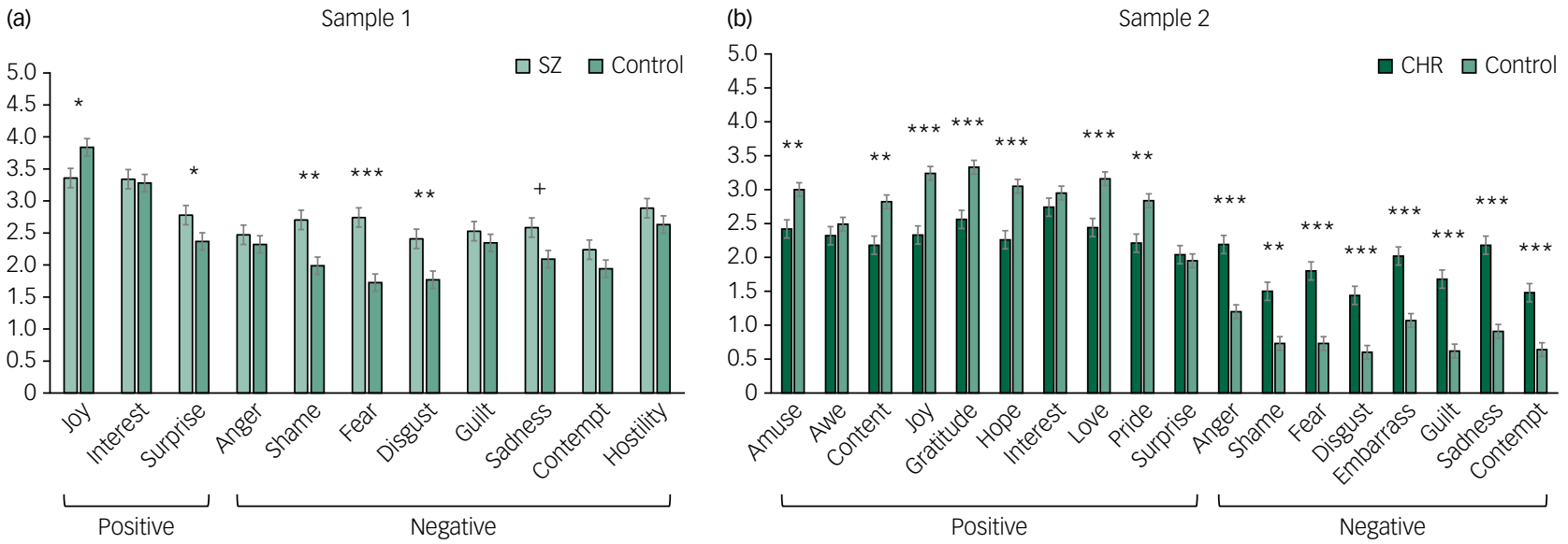

Fig. 2 Differences in discrete emotions in samples 1 and 2.

In sample 1, group differences for joy and surprise did not meet multiple testing correction criteria.

SZ, individuals with schizophrenia; CHR, individuals at clinical high risk for psychosis.

symptoms. Positive affect was positively associated with social functioning, and negative affect was negatively associated with negative role functioning. Higher levels of SIPS symptoms were all related to lower levels of social and role functioning (Fig. 3, right panel). All relationships remained unchanged after correcting for multiple correlation tests.

\section{Exploratory affect relationships to negative symptom subtypes in} individuals with schizophrenia and at CHR

To further explore the relationship between affect and negative symptoms in individuals with schizophrenia, negative symptoms were decomposed in two sets of analyses: the two subdimensions of expressivity and motivation, and the five NIMH domains. When examining the two subdimensions of negative symptoms, strong relationships between diminished motivation and both positive affect and negative affect emerged, whereas expressivity was only marginally related to positive affect and unrelated to negative affect. When examining negative symptoms using the NIMH domains, correlation analyses showed that positive affect was significantly negatively related to all NIMH domains except alogia, and only marginally related to blunted affect. The relationship between negative affect and the five domains seemed more complex, with negative affect being significantly related only to anhedonia and marginally related to alogia and asociality (supplementary Table 1 available at https://doi.org/10.1192/bjo.2019.64). All relationships remained unchanged after correcting for multiple correlation tests.

The same two-dimension approach for negative symptoms in individuals at CHR for psychosis showed that higher levels of both dimensions of negative symptoms (i.e. diminished expressivity and diminished motivation) were related to lower levels of positive affect and higher levels of negative affect. In analysis of the
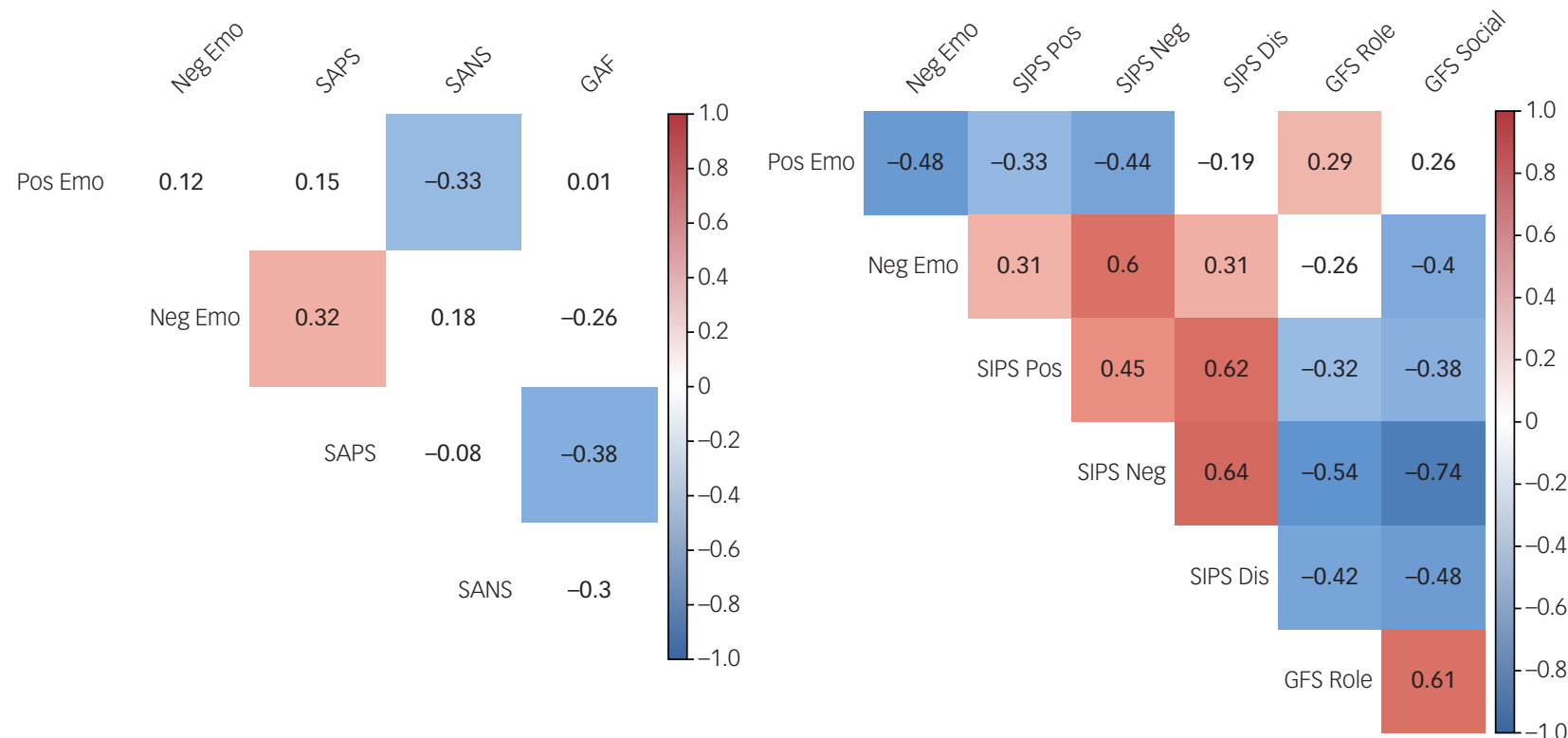

Fig. 3 Correlations between affect, clinical symptoms and functioning in samples 1 and 2.

Pos, positive; Neg, negative; Emo, affect; SAPS, Scale for the Assessment of Positive Symptoms; SANS, Scale for the Assessment of Negative Symptoms; GAF, Global Assessment of Functioning; SIPS, Structured Interview for Prodromal Syndromes; Dis, disorganized symptoms; GFS, Global Functioning Scale. 
individual SIPS items, every item was related to higher levels of negative affect, whereas positive affect was related only to the avolition, emotion expression and emotion experience items (supplementary Table 2). All relationships remained unchanged after correcting for multiple correlation tests.

\section{Secondary negative symptom influences on trait emotional experience}

Relationships between affect and negative symptoms controlling for anxiety, depression and antipsychotics in individuals with schizophrenia

After controlling for anxiety, depression and antipsychotic medication, only the relationship between positive affect and negative symptoms remained significant in individuals with schizophrenia. For the five NIMH domains, only the relationship between positive affect and the NIMH domains of avolition, anhedonia and asociality remained significant (supplementary Table 3). All relationships remained unchanged after correcting for multiple correlation tests.

Relationships between affect and negative symptoms controlling for anxiety and depression in individuals at CHR

After controlling for anxiety and depression, only the relationship between negative affect and diminished motivation remained significant in individuals at CHR for psychosis. Similarly, only the relationships between negative affect and the individual SIPS components remained significant when controlling for anxiety and depression (supplementary Table 4). All relationships remained unchanged after correcting for multiple correlation tests.

\section{Discussion}

In this study we observed that clinical samples of individuals with schizophrenia and individuals at CHR for psychosis showed differences from their respective controls in trait emotional experiences. Furthermore, differences in trait emotional experiences were closely linked to clinical symptoms and functional outcomes. This study was the first of its kind to examine differences in trait emotion across a wide set of emotions in individuals with schizophrenia and individuals at CHR. In individuals with schizophrenia, the relationship between the positive-emotion deficit and negative symptoms remained after controlling for secondary influences of depression and anxiety. In individuals at CHR, it is noteworthy that controlling for depression and anxiety eliminated the relationship between the positive-emotion deficit and negative symptoms. The following paragraphs discuss the implications of these findings.

\section{Levels of trait positive and negative affect in schizophrenia}

Individuals with schizophrenia reported higher levels of trait negative affect compared with individuals without schizophrenia, converging with prior literature, ${ }^{11,25}$ including findings by Cohen \& Minor, ${ }^{7}$ who showed that individuals with schizophrenia are likely to report aversive reactions to positive and negative stimuli. The present study showed that negative affect was driven by disgust, fear and shame in particular, converging with findings by Birchwood \& colleagues, ${ }^{70}$ who showed that fear and shame specifically may drive avoidance behaviour in psychosis (and disgust has similar avoidance functions). ${ }^{71}$ No differences were found for other negative emotions (i.e. anger, guilt, contempt and hostility) or for global positive affect in the groups with and without schizophrenia. However, examining specific positive emotions revealed preliminary opposing effects for joy (which was reduced) and surprise (which was elevated) in individuals with schizophrenia. Although these results did not stand up to multiple test correction, they directionally support existing evidence of reduced positive trait affect in individuals with schizophrenia. ${ }^{11,25,72}$ When interpreting the directionally elevated levels of surprise in individuals with schizophrenia, it is important to consider that individuals typically do not perceive surprise as emotionally positive but rather as ambiguous or even negative. $^{73}$

\section{Generalised emotion deficits in individuals at CHR}

Individuals at $\mathrm{CHR}$ for psychosis showed a broad array of abnormalities in trait positive and negative emotional experiences. Specifically, individuals at CHR reported lower levels of seven out of ten positive emotions (i.e. lower levels of amusement, contentment, joy, gratitude, hope, love and pride; no differences in awe, interest and surprise) and higher levels of all negative emotions compared with individuals not at CHR. These findings highlight that the emotion deficits in individuals at CHR are fairly generalised. It should be noted that exploratory analyses revealed that, for those at CHR who converted to a psychosis syndrome $(n=3$, from the original group of 50) during the 2-year follow-up period, emotional alterations were in the same direction as for the rest of the original group at $\mathrm{CHR}$, with directionally lower levels of positive emotions (mean 1.50, s.d. $=0.36$ ) and higher levels of negative emotions (mean 1.92, s.d. $=0.29$ ) compared with controls. Although the small number of individuals who developed a psychosis syndrome does not allow for direct comparisons with the general group at $\mathrm{CHR}$, this is an important question for future studies with larger samples.

\section{Discrete emotions versus affective valence}

Individuals with schizophrenia reported directionally different results for two of the three positive emotions (joy and surprise) and reported only some negative emotions as elevated. Although the differences in joy and surprise should be interpreted with caution, key significant findings remain (e.g. reports of higher levels of fear, shame and disgust, but not anger, guilt, contempt and hostility by individuals with schizophrenia compared with individuals without schizophrenia). These findings cumulatively emphasise the importance of moving beyond positive and negative affective valence toward considering discrete emotions (e.g. joy, surprise, fear, contempt), which may show unique patterns in individuals with schizophrenia and those at CHR for psychosis.

\section{Association of trait emotional experiences with symptoms and functioning}

Individuals with schizophrenia and individuals at CHR showed differences in relationships between trait emotional experiences on the one hand and clinical symptoms and functioning on the other. For individuals with schizophrenia, only positive affect was associated with negative symptoms, and only negative affect was associated with positive symptoms. These results support the stronger relationships reported in the literature between the positive-emotion deficit and negative symptoms. ${ }^{31,32,74}$ Reviews of negative emotions and negative symptoms yield unreliable associations between the two. ${ }^{11}$ Exploring specific aspects of negative symptoms suggested that the absence of a relationship between negative affect and negative symptoms may be partly explained by the fact that only diminished motivation was related to abnormalities in both positive and negative affect. Additionally, analysing negative symptoms at the level of the five domains of negative symptoms (anhedonia, avolition, asociality, alogia and blunted affect) showed that positive affect was most closely associated with all five, again supporting the notion that negative symptoms are those most closely tied to the positive affect deficit. Although the absence of some of these 
relationships may be due to the small sample, these findings support existing literature that positive emotions are most closely associated with negative symptoms. Unlike prior research, neither type of affect predicted decreased functioning. ${ }^{26,27}$ However, both studies used measures that differed from previous research, as we used self-report rather than interviewer ratings and assessed the presence/absence of specific skills rather making normative assessments of functioning.

In individuals at $\mathrm{CHR}$ for psychosis, trait emotional experiences were highly interconnected with symptoms and functioning. Both positive and negative affect were linked to both positive and negative symptoms. Moreover, both positive and negative affect were linked to both negative symptom dimensions (i.e. diminished expressivity; diminished motivation and pleasure). When breaking these symptoms down into the five subdomains, negative affect was interconnected with more subdomains than positive affect. These findings add important specificity for studies showing links between emotions and negative symptoms. ${ }^{5,37}$ The breadth of interconnectedness between trait emotion, symptoms and functioning highlights not only the generalised pathology of emotion abnormalities in the population at $\mathrm{CHR}$ for psychosis, but also the importance of making emotions the target of interventions for improving functional outcomes.

\section{Influence of secondary factors on trait emotional experience}

Secondary negative influences have not been studied in individuals with schizophrenia. This study found that controlling for secondary influences left only the relationship between positive affect and negative symptoms intact. The resilience of this relationship to these influences supports hypotheses that trait emotion differences, in particular positive-emotion deficits, may be more closely linked to the core of schizophrenia. ${ }^{9,75}$ Controlling for secondary influences in individuals at CHR for psychosis showed a strong relationship between negative affect and negative symptoms. These differences suggest that the emotion abnormalities (e.g. the positive-emotion deficit) seen in individuals at CHR may be driven more by a generalised psychopathology than the emotion abnormalities seen in individuals with schizophrenia. The concept of generalised psychopathology in individuals at CHR fits with recent findings that a high proportion of adolescents with anxiety and/or depression also display psychotic symptoms. ${ }^{76}$ It is possible that this high comorbidity rate in individuals at $\mathrm{CHR}$ reflects a close link between emotion dysregulation and psychosis early on. These findings also fit with other studies showing that individuals at CHR often report first with anxiety disorders or depression. ${ }^{17,77}$ The current findings suggest that a subset of individuals at CHR may have a high rate of generalised vulnerability for developing psychopathology. Identifying unique markers of these subgroups within the population of individuals at $\mathrm{CHR}$ can help isolate outcome groups earlier when young people first seek treatment.

\section{Limitations}

The present findings should be viewed in light of several limitations of the study. The group of individuals with schizophrenia was relatively small and it may thus have been underpowered to detect smaller effects. Nevertheless, the group with schizophrenia in the current study was larger than every group with schizophrenia in one review of studies on emotion differences in schizophrenia ${ }^{4}$ and larger than over two-thirds of groups in another review of trait emotion in schizophrenia. ${ }^{11}$ Additionally, direct comparisons were limited between the individuals with schizophrenia and those at CHR for psychosis, as two different versions of the scale for measuring trait emotions were used for the two groups.
Although exact parallels between the two groups cannot be drawn, both scales call attention to the importance of examining trait emotions at the discrete level, rather than always assuming generalisable effects for positive and negative affect. Literature showing that individuals with schizophrenia have a high degree of difficulty with emotional awareness suggests that caution should be used in interpreting strong differences between discrete emotions within the group with schizophrenia. ${ }^{17,78}$ However, the differences in some discrete emotions and not others found in the present study suggest that individuals with schizophrenia maintain some degree of differentiation. Future studies would benefit from examining how specific this level of differentiation is. Finally, differences in ages (e.g. adults with schizophrenia and adolescents and young adults at CHR) also limited direct comparisons between the two groups. However, the differences in age boost the external validity and generalisability of both samples.

\section{Future research}

Taken together, these findings suggest that trait emotion abnormalities are an important consideration in schizophrenia. Although the stability of trait emotional experience abnormalities has yet to be determined, such research is critical in determining whether trait affect should be the focus of psychosocial treatments such as cognitive-behavioural therapy for symptoms of the schizophrenia. Results also highlight the nuance that differentiating discrete emotions can add. Further, the present observations suggest that, although trait emotion abnormalities are implicated in both the prodrome and then later after onset, this critical domain may need to be assessed and conceptualised differently across stages of psychosis. Although examining trait emotion abnormalities in the prodrome is still in its early stages, early evidence highlights a hefty role for secondary influences. Experimental studies and large-scale longitudinal investigations will be instrumental in improving our understanding of this important area, and for driving early identification and treatment development efforts.

Claire I. Yee (D), PhD, Postdoctoral Fellow, Department of Psychology and School of Education and Social Policy, Northwestern University, USA; Gregory P. Strauss, PhD, Assistant Professor, Department of Psychology, University of Georgia, USA; Daniel N. Allen, PhD, Director of Clinical Training, Department of Psychology, University of Nevada, USA; Claudia M. Haase, PhD, Assistant Professor, School of Education and Social Policy, Northwestern University, USA: David Kimhy, PhD, Associate Professor, Department of Psychiatry, Icahn School of Medicine at Mount Sinai, USA; Vijay A. Mittal, PhD, Associate Professor, Department of Psychology, Northwestern University, USA

Correspondence: Claire Yee, Department of Psychology, Northwestern University, Swift Hall 102, 2029 Sheridan Road, Evanston, IL 60201, USA. Email: claire.yee@northwestern.edu

First received 20 Feb 2019, final revision 20 Jun 2019, accepted 12 Aug 2019

\section{Funding}

This work was supported by National Institutes of Health grants R01MH094650 and R21MH110374 to V.A.M, R21MH115231 to C.M.H. and V.A.M, and K23MH092530 to G.P.S

\section{Supplementary material}

Supplementary material is available online at https://doi.org/10.1192/bjo.2019.64.

\section{References}

1 Kraepelin E. Dementia Praecox and Paraphrenia (trans RM Barclay, ed GM Robertson). Chicago Medical Book Co, 1919.

2 Herbener ES, Song W, Khine TT, Sweeney JA. What aspects of emotional functioning are impaired in schizophrenia? Schizophr Res 2008; 98: 239-46. 
3 Kring AM, Elis O. Emotion deficits in people with schizophrenia. Annu Rev Clin Psychol 2013; 9: 409-33.

4 Kring AM, Moran EK. Emotional response deficits in schizophrenia: insights from affective science. Schizophr Bull 2008; 34: 819-34.

5 Phillips LK, Seidman LJ. Emotion processing in persons at risk for schizophrenia Schizophr Bull 2008; 34: 888-903.

6 Yan C, Cao Y, Zhang Y, Song LL, Cheung EF, Chan RC. Trait and state positive emotional experience in schizophrenia: a meta-analysis. PLoS One 2012; 7(7): e40672.

7 Cohen AS, Minor KS. Emotional experience in patients with schizophrenia revisited: meta-analysis of laboratory studies. Schizophr Bull 2008; 36: 143-50.

8 Llerena K, Strauss GP, Cohen AS. Looking at the other side of the coin: a metaanalysis of self-reported emotional arousal in people with schizophrenia. Schizophr Res 2012; 142: 65-70.

9 Strauss GP, Gold JM. A new perspective on anhedonia in schizophrenia. Am J Psychiatry 2012; 169: 364-73.

10 Edwards J, Jackson HJ, Pattison PE. Emotion recognition via facial expression and affective prosody in schizophrenia: a methodological review. Clin Psychol Rev 2002; 22: 789-832.

11 Horan WP, Blanchard JJ, Clark LA, Green MF. Affective traits in schizophrenia and schizotypy. Schizophr Bull 2008; 34: 856-74.

12 Kee KS, Horan WP, Salovey P, Kern RS, Sergi MJ, Fiske AP, et al. Emotional intelligence in schizophrenia. Schizophr Res 2009; 107: 61-8.

13 Kimhy D, Vakhrusheva J, Jobson-Ahmed L, Tarrier N, Malaspina D, Gross JJ. Emotion awareness and regulation in individuals with schizophrenia: implications for social functioning. Psychiatry Res 2012; 200: 193-201.

14 Yamauchi K, Aki H, Tomotake M, Iga Jl, Numata S, Motoki I, et al. Predictors of subjective and objective quality of life in outpatients with schizophrenia. Psychiatry Clin Neurosci 2008; 62: 404-11.

15 Fusar-Poli P, Bonoldi I, Yung AR, Borgwardt S, Kempton MJ, Valmaggia L, et al Predicting psychosis: meta-analysis of transition outcomes in individuals at high clinical risk. Arch Gen Psychiatry 2012; 69: 220-9.

16 Yung AR, Yuen HP, Berger G, Francey S, Hung TC, Nelson B, et al. Declining transition rate in ultra high risk (prodromal) services: dilution or reduction of risk? Schizophr Bull 2007; 33: 673-81.

17 Addington J, Cornblatt BA, Cadenhead KS, Cannon TD, McGlashan TH, Perkins $D$, et al. At clinical high risk for psychosis: outcome for nonconverters. Am J Psychiatry 2011; 168: 800-5.

18 Schmidt SJ, Schultze-Lutter F, Schimmelmann BG, Maric NP, Salokangas RKR Riecher-Rössler $A$, et al. EPA guidance on the early intervention in clinical high risk states of psychoses. Eur Psychiatry 2015; 30: 388-404.

19 Corcoran CM, Kimhy D, Parrilla-Escobar MA, Cressman VL, Stanford AD, Thompson J, et al. The relationship of social function to depressive and negative symptoms in individuals at clinical high risk for psychosis. Psychol Med 2011; 41: 251-61.

20 Velligan $\mathrm{DI}$, Gonzalez JM. Rehabilitation and recovery in schizophrenia. Psychiatr Clin North Am 2007; 30: 535-48.

21 Feldman Barrett $L$, Russell JA. Independence and bipolarity in the structure of current affect. J Pers Soc Psychol 1998; 74: 967-84.

22 Watson D, Clark LA, Tellegen A. Development and validation of brief measures of positive and negative affect: the PANAS scales. J Pers Soc Psychol 1988; 54: 1063.

23 Mineka S, Watson D, Clark LA. Comorbidity of anxiety and unipolar mood disorders. Annu Rev Psychol 1998; 49: 377-412.

24 Watson D, Clark LA, Harkness AR. Structures of personality and their relevance to psychopathology. J Abnorm Psychol 1994; 103: 18-31.

25 Suslow T, Roestel C, Ohrmann P, Arolt V. The experience of basic emotions in schizophrenia with and without affective negative symptoms. Compr Psychiatry 2003; 44: 303-10.

26 Blanchard JJ, Mueser KT, Bellack AS. Anhedonia, positive and negative affect, and social functioning in schizophrenia. Schizophr Bull 1998; 24: 413-24.

27 Cohen AS, Dinzeo TJ, Nienow TM, Smith DA, Singer B, Docherty NM. Diminished emotionality and social functioning in schizophrenia. J Nerv Ment Dis 2005; 193: 796-802.

28 Kentros MK, Terkelsen K, Hull J, Smith TE, Goodman M. The relationship between personality and quality of life in persons with schizoaffective disorder and schizophrenia. Qual Life Res 1997; 6: 118-22.

29 Gooding DC, Tallent KA. Spatial, object, and affective working memory in social anhedonia: an exploratory study. Schizophr Res 2003; 63: 247-60.

30 Horan WP, Blanchard JJ. Emotional responses to psychosocial stress in schizophrenia: the role of individual differences in affective traits and coping. Schizophr Res 2003; 60: 271-83.

31 Horan WP, Green MF, Kring AM, Nuechterlein KH. Does anhedonia in schizophrenia reflect faulty memory for subjectively experienced emotions? J Abnorm Psychol 2006; 115: 496-508.
32 Watson D, Naragon-Gainey K. On the specificity of positive emotional dysfunction in psychopathology: evidence from the mood and anxiety disorders and schizophrenia/schizotypy. Clin Psychol Rev 2010; 30: 839-48.

33 Blanchard JJ, Squires D, Henry T, Horan WP, Bogenschutz M, Lauriello J, et al. Examining an affect regulation model of substance abuse in schizophrenia: the role of traits and coping. J Nerv Ment Dis 1999; 187: 72-9.

34 Couture S, Lecomte T, Leclerc $\mathrm{C}$. Personality characteristics and attachment in first episode psychosis: impact on social functioning. J Nerv Ment Dis 2007; 195: 631-9.

35 Horan WP, Subotnik KL, Reise S, Ventura J, Nuechterlein KH. Personality characteristics in recent-onset schizophrenia: Iongitudinal stability and clinical correlates. Psychol Med 2005; 35: 995-1005.

36 Lysaker PH, Taylor AC. Personality dimensions in schizophrenia: associations with symptoms and coping concurrently and 12 months later. Psychopathology 2007; 40: 338-44.

37 Seo E, Bang M, Lee E, An SK. Aberrant tendency of noncurrent emotional experiences in individuals at ultra-high risk for psychosis. Psychiatry Investig 2018; 15: 876-83.

38 Kirkpatrick B, Buchanan RW, Ross DE, Carpenter WT. A separate disease within the syndrome of schizophrenia. Arch Gen Psychiatry 2001; 58: 165-71.

39 Kirkpatrick B, Galderisi S. Deficit schizophrenia: an update. World Psychiatry 2008; 7: 143-7

40 Strauss GP, Allen DN, Duke LA, Ross SA Schwartz J, Automatic affective processing impairments in patients with deficit syndrome schizophrenia. Schizophr Res 2008; 102: 76-87.

41 Strauss GP, Allen DN, Ross SA, Duke LA, Schwartz J. Olfactory hedonic judgment in patients with deficit syndrome schizophrenia. Schizophr Bull 2009; 36: $860-8$

42 Strauss GP, Jetha SS, Ross SA, Duke LA, Allen DN. Impaired facial affect labeling and discrimination in patients with deficit syndrome schizophrenia. Schizophr Res 2010; 118: 146-53.

43 Piskulic D, Addington J, Cadenhead KS, Cannon TD, Cornblatt BA, Heinssen R, et al. Negative symptoms in individuals at clinical high risk of psychosis. Psychiatry Res 2012; 196: 220-4.

44 Pelletier-Baldelli A, Strauss GP, Visser KH, Mittal VA. Initial development and preliminary psychometric properties of the Prodromal Inventory of Negative Symptoms (PINS). Schizophr Res 2017; 189: 43-9.

45 Azar M, Pruessner M, Baer LH, Iyer S, Malla AK, Lepage M. A study on negative and depressive symptom prevalence in individuals at ultra-high risk for psychosis. Early Interv Psychiatry 2018; 12: 900-6.

46 Goetz $\mathrm{T}$, Frenzel AC, Stoeger $\mathrm{H}$, Hall NC. Antecedents of everyday positive emotions: an experience sampling analysis. Motiv Emotion 2010; 34: 49-62.

47 Pressman SD, Jenkins BN, Kraft-Feil TL, Rasmussen H, Scheier MF. The whole is not the sum of its parts: specific types of positive affect influence sleep differentially. Emotion 2017; 17: 778-93.

48 Stellar JE, John-Henderson N, Anderson CL, Gordon AM, MCNeil GD, Keltner D. Positive affect and markers of inflammation: discrete positive emotions predict lower levels of inflammatory cytokines. Emotion 2015; 15: 129-33.

49 Blanchard JJ, Cohen AS. The structure of negative symptoms within schizophrenia: implications for assessment. Schizophr Bull 2005; 32: 238-45.

50 Ahmed AO, Kirkpatrick B, Galderisi S, Mucci A, Rossi A, Bertolino A, et al. Crosscultural validation of the 5 -factor structure of negative symptoms in schizophrenia. Schizophr Bull 2018; 46: 3383-95.

51 Strauss GP, Esfahlani FZ, Galderisi S, Mucci A, Rossi A, Bucci P, et al. Network analysis reveals the latent structure of negative symptoms in schizophrenia. Schizophr Bull 2018; Sep 26: doi 10.1093/schbul/sby133 [Epub ahead of print].

52 Strauss GP, Nuñez A, Ahmed AO, Barchard KA, Granholm E, Kirkpatrick B, et al. The latent structure of negative symptoms in schizophrenia. JAMA Psychiatry 2018; 75: 1271-9.

53 American Psychiatric Association. Diagnostic and Statistical Manual of Mental Disorders (4th edn text revision) (DSM-IV-TR). APA 2000.

54 First MBSpitzer RLGibbon MWilliams JB. Structured Clinical Interview for DSMIV Axis I Disorders - Clinician Version (SCID-I/CV). American Psychiatric Press, 1996.

55 Andreasen NC. Scale for the Assessment of Positive Symptoms (SAPS). University of Iowa, 1984.

56 Andreasen NC. Scale for the Assessment of Negative Symptoms (SANS): conceptual and theoretical foundations. Br J Psychiatry 1989; 155: 53-8.

57 Overall JE, Gorham DR. The Brief Psychiatric Rating Scale. Psychol Rep 1962; 10: 799-812.

58 Izard C, Libero D, Putnam P, Haynes O. Stability of emotion experiences and their relations to traits of personality. J Pers Soc Psychol 1993; 64: 847-60.

59 Izard CE. The Face of Emotion. Appleton-Century-Crofts, 1971. 
60 Miller TJ, McGlashan TH, Rosen JL, Cadenhead K, Ventura J, McFarlane W, et al. Prodromal assessment with the Structured Interview for Prodroma Syndromes and the Scale of Prodromal Symptoms: predictive validity, interrater reliability, and training to reliability. Schizophr Bull 2003; 29: 703-15.

61 McGlashan T, Walsh BC, Woods S. The Psychosis-Risk Syndrome: Handbook for Diagnosis and Follow-Up. Oxford University Press, 2010

62 Beck AT, Steer RA, Brown GK. Manual for the Beck Depression Inventory-II. Psychological Corporation, 1996.

63 Beck AT, Epstein N, Brown G, Steer RA. An inventory for measuring clinical anxiety: psychometric properties. J Consult Clin Psychol 1988; 56: 893-7.

64 Cornblatt BA, Auther AM, Niendam T, Smith CW, Zinberg J, Bearden C, et al. Preliminary findings for two new measures of social and role functioning in the prodromal phase of schizophrenia. Schizophr Bull 2007; 33: 688-702.

65 Fredrickson $\mathrm{BL}$, Tugade MM, Waugh CE, Larkin G. What good are positive emotions in crises? A prospective study of resilience and emotions following the terrorist attacks on the United States on September 11th, 2001. J Pers Soc Psychol 2003; 84: 365-76.

66 Strauss GP, Horan WP, Kirkpatrick B, Fischer BA, Keller WR, Miski P, et al. Deconstructing negative symptoms of schizophrenia: avolition-apathy and diminished expression clusters predict clinical presentation and functional outcome. J Psychiatr Res 2013; 47: 783-90.

67 Azis M, Strauss GP, Walker E, Revelle W, Zinbarg R, Mittal V. Factor analysis of negative symptom items in the Structured Interview for Prodromal Syndromes. Schizophr Bull 2018; Dec 8: doi 10.1093/schbul/sby177 [Epub ahead of print].

68 Kirkpatrick B, Fenton WS, Carpenter WT, Marder SR, The NIMH-MATRICS consensus statement on negative symptoms. Schizophr Bull 2006; 32: 214-19.

69 Benjamini $Y$, Hochberg Y. Controlling the false discovery rate: a practical and powerful approach to multiple testing. J R Stat Soc B 1995: 289-300.

70 Birchwood M, Meaden A, Trower P, Gilbert P, Plaistow J. The power and omnipotence of voices: subordination and entrapment by voices and significant others. Psychol Med 2000; 30: 337-44.
71 Rozin P, Fallon AE. A perspective on disgust. Psychol Rev 1987; 94: 23-41.

72 Hooker $\mathrm{Cl}$, Benson TL, Gyurak A, Yin $\mathrm{H}$, Tully LM, Lincoln SH. Neural activity to positive expressions predicts daily experience of schizophrenia-spectrum symptoms in adults with high social anhedonia. J Abnorm Psychol 2014; 123: 190-204.

73 Lorini E, Castelfranchi C. The cognitive structure of surprise: looking for basic principles. Topoi 2007; 26: 133-49.

74 Freeman D, Garety PA. Connecting neurosis and psychosis: the direct influence of emotion on delusions and hallucinations. Behav Res Ther 2003; 41: 923-47.

75 Cohen AS, Najolia GM, Brown LA, Minor KS. The state-trait disjunction of anhedonia in schizophrenia: potential affective, cognitive and social-based mechanisms. Clin Psychol Rev 2011; 31: 440-8.

76 Wigman JT, van Nierop M, Vollebergh WA, Lieb R, Beesdo-Baum K, Wittchen HU, et al. Evidence that psychotic symptoms are prevalent in disorders of anxiety and depression, impacting on illness onset, risk, and severity: implications for diagnosis and ultra-high risk research. Schizophr Bull 2012; 38: 247-57.

77 Velthorst E, Nieman DH, Becker HE, van de Fliert R, Dingemans PM, Klaassen R et al. Baseline differences in clinical symptomatology between ultra high risk subjects with and without a transition to psychosis. Schizophr Res 2009; 109: $60-5$

78 Van't Wout M, Aleman A, Bermond B, Kahn RS. No words for feelings: alexithymia in schizophrenia patients and first-degree relatives. Compr Psychiatry 2007: 48: 27-33. 